\title{
Status of Referral to Physiotherapy Among HIV Positive Patients at Chris Hani Baragwaneth Hospital Johannesburg South Africa 2005
}

\begin{abstract}
HIV continues to be a major health problem in South Africa. The multiple diagnoses that the disease presents with, needs a holistic and comprehensive management approach. Physiotherapy and rehabilitation play a role in this management approach. Understanding the full scope of conditions that are present and those that are suitable for physiotherapy intervention is an essential prerequisite to developing appropriate curricula, intervention models or systems. It is accepted that HIV rehabilitation interventions are based largely on functional deficits (O'Dell 1996), however in South Africa functional deficits have not been fully explored. A common

Myezwa $\mathrm{H}^{1}$, Stewart A $^{1}$, Mbambo N', Nesara $\mathrm{P}^{2}$.

1 Physiotherapy Department; University of the Witwatersrand, 7 York Road, Parktown, Johannesburg, South Africa.

2 Epidemiology Data Centre; University of the Witwatersrand, 7 York Road, Parktown, Johannesburg, South Africa. starting point, with the medical model of management was considered to be at the diagnosis level as this information would be more readily available than functional deficits.

Purpose: This study aimed to establish how much and in which aeitiology is physiotherapy involved in the management of HIV within an inpatient hospital setting at Chris Hani Baragwaneth Hospital. This minor study forms part of a larger study establishing physiotherapy curricula needs.

Method: A retrospective review of patient records was carried out in order to identify conditions suitable for physiotherapy and to determine the referral patterns to physiotherapy.

Findings: Of the 732 records reviewed and used in the study, $47 \%(n=344)$ of the patients were HIV positive. From these records, $19 \%(n=139)$ had diagnoses considered suitable for physiotherapy and only 2\% $(n=3)$ of these 139 patients were referred to physiotherapy.

Conclusion: Almost half of the patients in the medical units were HIV positive. Although the referral rate was very low, some of these patients presented with diagnosis that are traditionally seen by physiotherapists. None of the patients' records indicated examination of the patients' physical status such as exercise tolerance, mobility, muscle strength, lung function or pain. This study is by no means fully representative of the full scope of the epidemiology of conditions that can be seen by physiotherapists or their referral status but does give some indication of what conditions are, and could potentially interface with physiotherapy.
\end{abstract}

KEY WORDS: HIV, PHYSIOTHERAPY, REFERRAL, CONDITIONS.

\section{INTRODUCTION}

The antiretroviral programme in South Africa was launched in 2003 (DOH 2004 ). Although it is considered as far from adequate (TAC 2005) it is slowly expanding and prolonging life and improving the survival rates of people who are infected ( Lawn et al, 2005 ). In South Africa, rates of admission into hospital for people living with AIDS remains high (Reid 2005) and people living with AIDS present with multisystem disorders resulting in different diagnoses and a wide range of problems. Some of the problems have been found to benefit from physiotherapy and other rehabilitation interventions. The problematic areas documented are respiratory, neurological, musculo-skeletal and painful syndromes (McClure 1993). The specific roles played by physiotherapy within the HIV/AIDS pandemic in Africa have not been explored. It is against this background that this study describes how much interaction there is between physiotherapy and HIV positive patients in an inpatient setting as part of a larger study which is establishing the curricula needs of physiotherapy in HIV and seeks to determine the range of conditions that present in a cohort of patients admitted to hospital with HIV.

\section{CONDITIONS WITH MANIFESTATIONS COMMONLY SEEN BY REHABILITA- TION PROFESSIONALS}

Three organ systems, namely the respiratory, neurological and musculoskeletal are found to be commonly affected by HIV and result in disorders that may need rehabilitation.

Pneumonias caused by various opportunistic organisms are a common presenting feature of AIDS (Johnson 1985) with tuberculosis being one of the most common opportunistic infection associated with HIV. Out of 42 million

\section{CORRESPONDENCE TO:}

\section{Myezwa $\mathrm{H}$}

Physiotherapy Department;

University of the Witwatersrand,

7 York Road, Parktown,

Johannesburg, South Africa.

Tel: (011) 717-3721

Email: hellen.myezwa@wits.ac.za 
people infected by HIV 15 million are co-infected with TB (McShane 2005).

HIV has been reported to be particularly neurotrophic. At seroconversion phase, neurological-like symptoms have been reported e.g. aseptic meningitis, brachial neuritis and cauda equine syndrome. Gillain Barre syndrome has also been described at seroconversion and at the asymptomatic immuno-competent phase. Other neurological conditions reported include the "younger stroke" meningitic and encephalitic disorders (Manji and Millar 2004) and peripheral neuropathies are emerging with the introduction of ARVs. (Mc Aurther 2004).

According to Tehranzedah et al (2004) some of the musculoskeletal conditions that develop in HIV infected clients include septic bursitis, septic arthritis, pyogenic osteomyelitis and tuberculous infections, infection of the skin and subcutaneous tissue resulting in cellulites and soft tissue abscesses. In the USA septic arthritis is reported as the most common musculoskeletal infection in HIV (Tehranzedah et al 2004). Many of the conditions present with non specific symptoms of myalgia, pain, fever and fatigue. (Tehranzadeh et al 1994). To manage HIV effectively the rehabilitation professional should be familiar with the aetiology of the disease. (O Connell P. G, Levinson S. F. 1991).

A more accurate picture of the etiology of disease that physiotherapists should understand would be constructed by looking at patients already referred for rehabilitation. This option was explored at $\mathrm{CHBH}$ and it was found that patients presenting for treatment in the physiotherapy department did not always have their HIV status revealed.

\section{PHYSIOTHERAPY IN HIV}

The role of physiotherapy in other parts of the world has been described (Nixon 2000, Canadian working group on HIV and rehabilitation 2000, McClure 1993, O’Dell 1990,Galatinho 1991) and physiotherapy intervention is considered important before, during and after hospital admission for alleviating disuse atrophy and cardiovascular de-conditioning (Mc Clure 1993, Lang 1993). In peripheral neuropathy it has been shown that through behavioural methods which may be interpreted as physical activity and physiotherapy, pain can be reduced and the health-related quality of life
(HRQoL) of the HIV patient can be improved (Germaine et al 2005). Aerobic exercise and fitness have in general, positive impact at all stages of HIV disease (Nixon et al 2005, Mars 2004). Progressive resisted exercises ( PRE) in seven studies reviewed by O Brien et al (2005) in a Cochrane review, showed significant increases in patients' body weight, body composition strength outcomes, health related QOL and cardiopulmonary status. Physical activity, specific exercises, relaxation, assessment, treatment and management of functional problems and management of pain are among those interventions that have been used and documented. Various physiotherapy interventions are reported to have a positive impact on the different diagnoses with which patients with HIV present (O' Dell 1992) but some of these intervention strategies continue to be explored for effectiveness (Roubenoff 2002, Mc Clure, 1993 , Galantinho, 1988).

In South Africa the range of patient diagnoses among people living with AIDS treated by physiotherapists is not documented. The Canadian working group on HIV and rehabilitation (CWGHR)have stated the importance of establishing what conditions are seen by physiotherapists as an important prerequisite to developing services ( CWGHR 2000) No literature could be found to outline which conditions commonly seen in other countries are also seen by therapists in South Africa. To that effect, this study reviewed the records of patients admitted into the medical unit at Chris Hani Baragwanath hospital (CBH) using the method outlined hereunder.

\section{METHODOLOGY}

Although Lang (1993) found that therapeutic intervention is based more on examination findings than on the medical diagnoses, this study sought to look at the diagnoses that are commonly seen by physiotherapists among an HIV population and examined the notes for recorded impairments and functional deficits and established if referral took place.

\section{PROCEDURE}

- The records of patients who were referred to the medical units between June 2004 and June 2005 were identified physically by the records officer and 732 records were identified for review in this study.

- Two reviewers, both qualified physiotherapists with clinical experience identified files for patients who were HIV positive and had diagnoses that were frequently referred for physiotherapy. These files were categorized into five areas:

(a) HIV positive but died

(b) Files with no information

(c) Files with other diagnoses not HIV related

(d) HIV positive with diagnoses that were suitable for physiotherapy

(e) HIV positive with diagnoses that were unsuitable for physiotherapy

Using both the literature and experience, the reviewers set up inclusion criteria for classifying, coding and ranking common diagnoses. Diagnoses that were found were pneumonia, post TB bronchiectasis, TB with coinfection, neuromuscular conditions including Gullain Barre Syndrome, cerebral vascular accidents and polyneuropathies.

Case files that were reviewed as suitable for physiotherapy were isolated for further review to establish:

- Age and gender of the patient,

- The diagnosis that was identified as suitable for physiotherapy,

- Whether the patient was referred to physiotherapy,

- Whether the common signs and symptoms (Impairments) that are normally referred for physiotherapy such as. weakness of the musculoskeletal system, state of ambulation and increased secretion production were present and

- The scope of tests that were conducted

Case files with single diagnosis unsuitable for physiotherapy such as gastroenteritis, renal disease TB with no productive cough or coinfection and with only retro viral disease RVD. offered as diagnosis were excluded.

\section{DATA ANALYSIS}

Descriptive statistics were used to determine the distribution of the different impairment categories on files that met the inclusion criteria. Frequencies and tables were used to present the distribution of demographic characteristics, diagnoses and referral status of the patients. 


\section{RESULTS}

Table 1 shows how the files were distributed according to the categories.

\section{Demographic characteristics}

Of the 139 patients who were considered potential candidates suitable for physiotherapy assessment, seven had undocumented information such as gender, age or clear HIV status in their files and were thus excluded from the study.

Table 1: Illustration of distribution of files according to categories $(n=732)$.

\begin{tabular}{|l|l|}
\hline File Categories & $\%$ (n) \\
\hline No information in files & $5(37)$ \\
\hline HIV negative with other diagnosis & $53(388)$ \\
\hline HIV positive but died & $12(88)$ \\
\hline HIV positive with diagnosis unsuitable for physiotherapy referral & $11(80)$ \\
\hline HIV positive with diagnosis for possible physiotherapy referral & $16(117)$ \\
\hline Tuberculosis and HIV* & $3(22)$ \\
\hline
\end{tabular}

*Tb was extracted as an addional category

Of the 732 patient records, 139 (19\%) had diagnoses considered suitable for physiotherapy. These are the record that were further reviewed.

Table 3: Illustration of diagnoses found in patients' records $(\mathrm{N}=132)$.

\begin{tabular}{|l|l|l|}
\hline Rank & Diagnosis & $\mathbf{N}(\%)$ \\
\hline 1 & Pneumonia 39 & $39(29)$ \\
\hline 2 & Meningitis & $34(25)$ \\
\hline 3 & Tuberculosis 27 (20) & \\
\hline 4 & Lower respiratory tract infection 26 (19) & \\
\hline 5 & Meningitis and cryptococcal meningitis 25 (18) & \\
\hline 6 & Lower motor neuron paralysis 11 (8) & $8(6)$ \\
\hline 7 & Retro-Viral Disease ( with no specific diagnosis)*1 & $8(6)$ \\
\hline 8 & Thrombocytopenia( seen in combination with other conditions) & $178^{*}$ \\
\hline Total & & \\
\hline
\end{tabular}

* The total exceeds 132 because some patients had more than one diagnosis

*1 Although this means a diagnosis of HIV, in the notes this was given as the patients diagnosis when the patient was deconditioned with multiple non specific impairments such as aneamia, and weight loss in combination with another diagnosis such as meningitis or pneumonia. This was confirmed by the head of the medical unit.

Table 4: Ranking of Diagnosis combinations.

\begin{tabular}{|l|l|l|}
\hline Ranking & Diagnosis & N (\%) \\
\hline 1 & Pneumonia & $22(16)$ \\
\hline 2 & Meningitis & $11(8)$ \\
\hline 3 & PCP and TB & $9(7)$ \\
\hline 4 & PCP & $8(6)$ \\
\hline 5 & PCP and LRTI & $7(5)$ \\
\hline 6 & $\begin{array}{l}\text { TB and Pneumonia 5 (4) 8 Pneumonia and LRTI,LRTI } \\
\text { and TB, lung abscess }\end{array}$ & $3(2)$ \\
\hline
\end{tabular}

Table 2: Age-group distribution of patients $(n=132)$.

\begin{tabular}{|l|l|}
\hline Age range & $\mathbf{N}(\%)$ \\
\hline $15-19$ & $5(4)$ \\
\hline $20-24$ & $12(9)$ \\
\hline $25-29$ & $25(19)$ \\
\hline $30-34$ & $29(22)$ \\
\hline $35-39$ & $19(14)$ \\
\hline $40-44$ & $15(11)$ \\
\hline $45-49$ & $15(11)$ \\
\hline $50-54$ & $7(5)$ \\
\hline $55-72$ & $6(5)$ \\
\hline Total & $132(100)$ \\
\hline
\end{tabular}

The majority of the patients were in the age group, 30-34.

\section{Types of diagnoses}

The most common diagnoses were ranked as shown in table 3 . The most frequent diagnoses were ranked as 1 while the least common was ranked a 8 .

The two most frequent diagnoses were those with respiratory (ranked as 1, 3,\&4) and neurological problems (ranked 2,5,6).

Some diagnoses presented as a single diagnosis or in combination with others.

Table 4 shows the different combinations of diagnoses ranked in order of frequency as seen per patient.

Although twenty one different diagnoses were identified in total, this table shows the top eight conditions. Respiratory conditions such as pneumonia and other combinations of different respiratory conditions occurred most frequently. The other conditions that were present in this cohort but are not ranked include post TB bronchiectasis, pleuritic effusion, pneumothorax, lower motor neuron paralysis, dementia, DVT, encephalitis, thrombocytopenia Ca brain, asthma and congestive cardiac failure.

\section{Referral Status of patients suitable for physiotherapy}

Only $2 \%(n=3)$ of the total number of patients further reviewed were referred and seen by physiotherapists while $98 \%$ $(n=129)$ were not referred. Ten $(7 \%)$ of the cases were discharged while non ambulant. These ten patients had a mean age of 37year (range 27-46), six were 
male, four were female and 2 of them had been referred for physiotherapy. One was diagnosed with peripheral neuropathy and CCF while the other had a lung abscess.

The other eight non ambulant patients had the following diagnosis individually or in combination with other diagnosis:

- Hemiparesis = 1

- Meningitis/ cryptococcal meningitis $=4$

- Lower motor neuron paralysis and polyneuropathy $=2$

- Lower respiratory tract infection $=3$

- Tuberculosis = 1

- Pneumonia and or PCP $=2$

- The third patient referred for physiotherapy had a lower respiratory tract infection and was ambulant.

\section{DISCUSSION}

The authors acknowledge that a prospective or larger cross sectional study examining functional deficits would build a better picture. However establishing a clear picture of the current situation will contribute to a more appropriate education or intervention model.

As the nature of HIV changes from being a terminal to a chronic illness, it is important to examine some of the interventions traditionally included in the management of chronic illness. Rehabilitation and physiotherapy are among some of the key interventions for dealing with the disabling impacts of chronic illness or conditions (Worthington 2005). However, differential diagnosis is seen by Lang (1993), in her experience with HIV, as essential for medical management and less so for rehabilitation. This sentiment is supported by Nixon and Cott (2000) who argued that the medical model lacks the conceptual framework to guide education research or practice in rehabilitation. Literature on rehabilitation described earlier and current medical practice is grounded in this same medical model (O'Dell, 1996 and 1998). Since there are no studies that have attempted to describe the population of HIV clients that may benefit from physiotherapy in the South African situation, this study was therefore considered an appropriate starting point in trying to establish what is being referred and seen by physiotherapists in spite of the paucity of information on functional and participation deficits.
The total number of HIV admissions to the hospital could not be obtained from the hospital information system at Chris Hani Baragwanath Hospital at the time of this study. However $42 \%$ of the total records reviewed were of patients who were HIV positive. In this cohort there was a higher number of female patients $57 \%(\mathrm{n}=76)$ with their mean age lower than that of males. This concurs with other literature (Rehle et al, 2003) where within the affected population females are in the majority. This should be noted by therapists for appropriate age and gender sensitive interventions within the continuum of care model of prevention, treatment and care (UNAIDS 2003). Furthermore, the number of patients with HIV still remains higher in the productive age group between 25 and 49, the highest percentage being in the 30-34 age range. This is consistent with statistics released nationally (Rehle et al 2003) and internationally (WHO 2004)..

O' Dell (1993) conducted a retrospective study in a Philadelphia teaching hospital on medical records of persons hospitalized with complications of HIV and referred to rehabilitation. The study did not indicate the total number of records reviewed. Case notes reviewed showed a wide range of admitting diagnoses including similar ones found among the patient notes reviewed at $\mathrm{CHBH}$. Some of the common diagnoses between O' Dell's (1993) study and this study are pneumonia, cryptococcal meningitis. Furthermore, assessments of patients with HIV done by O'Connell and Levinson (1991) in full on first reference and O`Dell (1993) found different functional deficits and impairments among patients with HIV. Some of the deficits included: pain, physical deconditioning, proximal and distal muscle weakness, paresis and immobility. In addition, this study revealed other diagnoses including tuberculosis, lower respiratory tract infections and lower motor neuron paralysis. This result reflects the existence of a need for physiotherapy among people living with HIV/AIDS (PLWA) because this range of diagnosis that were identified are commonly known to result in activity limitations that can be countered by physical therapy and rehabilitation interventions. In the cases reviewed by O'Dell $86.7 \%$ were referred to occupa- tional therapy and $80 \%$ to physiotherapy. In this study only 3 patients $(2 \%)$ were referred for physiotherapy. In comparison with referral in western countries this figure is very low indicating that there is no focus on the client's physical and functional needs and subsequent quality of life. It is difficult though to draw a firm conclusion from the result as HIV related physical disability was not captured in the patient notes or on the discharge summary.

Although the general impact of exercise, as a modality of treatment for HIV patients has been shown in a Cochrane review(O'Brien 2005), to benefit people living with HIV, exercise is not considered as an option at Chris Hani Baragwanath hospital as indicated by the low referral rate of patients to physiotherapy. This may be attributed to this sample of patients being in the advanced stages of HIV as they were taken from patients that present themselves to the hospital and have been deemed ill enough to be admitted. This was confirmed by the head of the medical unit at $\mathrm{CHBH}$. where it was revealed that most patients are admitted for medical stabilization and are discharged as soon as this is achieved. All those who were reviewed for referral were discharged home with no functional assessment evident. It is difficult to draw firm conclusions on their need for rehabilitation if there is no detail of their impairment status other than laboratory markers, and general status and no further assessment is available of their physical and functional status. If patients are not referred for further assessment and physiotherapy it can be concluded that many patients with HIV related complications that can benefit from physiotherapy are not receiving a comprehensive model of care due to this oversight.

\section{LIMITATIONS}

The researcher accepts that the direct need for physiotherapy cannot be determined from diagnosis alone however known aetiology does give an indication of the known impairments and subsequent disability. The use of the diagnosis may limit the accuracy of the true picture of which conditions may need physiotherapy but this is essentially the only common starting point that gives adequate overlap with the medical teams 
focus in the management of patients. To minimize this limitation we examined the case notes closely and isolated any impairment such as increased secretion production and muscle strength. Further research should endeavor to confirm the diagnosis, impairments and activity limitations.

Other limitations should also be considered: First as patients notes were selected from the medical units, many, of the patients had advanced disease as indicated in general by the head of unit and their low CD4 counts. Therefore this may give a skewed picture of the referral status. However except for those who died and were excluded all patients analysed for physiotherapy referral were discharged home. Other units such as neurology should be assessed for referral patterns to complete the picture. Another limitation is that information was retrospectively collected from the case notes whereas a prospective study would be more valid. The notes were standard in structure but there was variation in the format and the information was given by different doctors thus leaving room for error.

\section{CONCLUSION}

Despite such a high proportion of patients admitted to the medical wards at Chris Hani Baragwanath Hospital South Africa being HIV positive, many patients living with AIDS are not seen by rehabilitation professionals in the hospital setting. There is little or no referral of patients or first line contact with physiotherapy or other traditional rehabilitation professionals. There is much to be explored and understood in the area of rehabilitation services for people living with AIDS within the South African context. Rehabilitation has the ability to impact on a number of domains across the full range of body function and structure, activity and participation and to contribute to better integrated care. The physiotherapists' role in increasing exercise tolerance, muscle strength, (O'Brien 2005) improving general body conditioning ( $\mathrm{O}$ 'Dell 1993), teaching pain ( Germain et al 2005) management strategies, improving function and activity and treating stress and anxiety related symptoms (Lang, 1993) is not fully exploited. This study did not investigate the underlying reasons for the lack of referral or the lack of active participation of rehabilitation professionals. A more accurate picture of the needs is required, therefore it is important for further studies to examine patients with HIV and document the different deficits with which they present. The results from such a study may assist in informing curriculum policy and programme development for PLWA in institutional, hospital and community settings.

From this study, we recommend that medical practitioners should be encouraged to screen patients for function and activity limitations, and where necessary refer them for further assessment by the rehabilitation team. Physiotherapists should be encouraged to market their services and educate other professionals on their role in managing HIV and AIDS patients. In order to do this, further research is needed to establish what functional deficits exist and exactly where the needs lie.

\section{REFERENCES}

Antoni MH, Bagget L, Ironson G, 1991. Behavioural stress management intervention buffers distress responses and immunological changes following notification of HIV-1 seropositivity. Journal of Consult Clinical Psychology: 59 (6) : 906-15

The Canadian Working Group on HIV and rehabilitation, 2000. Policy issues on rehabilitation in the context of HIV disease: A background and Position paper; www,hivand rehab.ca

Galantinho M., 1987. An overview of the AIDS patient. Clinical Management 7:12-13

Galantinho ML, Mukand J, Freed MM,1991. Physical therapy management of patients with HIV infection in Mukand J (ed) Rehabilitation for Patients with HIV disease. New York, McGraw Hill,

Germaine A Griswold, Evans S., Spielman L. and Fishman B., 2005. Coping Strategies of HIV patients with peripheral neuropathy. AIDS Care; 711-720

Johnson MCI Pneumonia in Acquired Immune Deficiency Syndrome. BMJ Vol 290 1299-1301

Lang C. 1993, Experience of community physiotherapy for people with HIV infection, British Journal of Occupational Therapy; 56: 6

Lawn SD, Landon M, Orrell C, Bekker L, Wood R, 2005. Early mortality among adults accessing a community-based antiretroviral service in South Africa: implications for programme design AIDS. Dec 2;19 (18);2141-8.

Manji H, Millar R. 2004. The neurology of AIDS, Journal of Neurology , Neurosurgery and Psychiatry 75:i29
Mc Authur J.C.,2005. John Hopkins HIV Report www.hopkins.aids

Mc Clure J. 1993. The Role of Physiotherapy in HIV and AIDS. Physiotherapy, 79 (6) 388393

Mc Shane H. 2005. Coinfection with HIV and TB Double Trouble. International journal of STDs and AIDS: 16:95-101

Nixon S, Brien K, Glazier R H, Tynan AM. 2005. Aerobic Exercise interventions for adults living with HIV;2: The Cochrane Collaboration Wiley \&Sons.

Nixon S. Cott C.A. 2000. Shifting Perspectives: Reconceptualizing HIV Disease in a Rehabilitation Framework. Physiotherapy, Canada; 52 189-197

O Brien K, Nixon S, Glazier RH Tynan AM ,2005. Progressive resistive exercise interventions for adults living with HIV/AIDS. Cochrane Collaboration 2 Wiley Publishers.

O Connell P. G, Levinson S. F.,1991. Experience with rehabilitation in acquired immunodeficiency syndrome. American Journal of Medical Rehabilitation; 70: 4 195-200

O Dell MW. 1993. Rehabilitation Medicine consultation in Persons Hospitalized with AIDS An analysis of 30 cases. Am J Phys Med Rehabilitation 72; 90-96

O’ Dell MW, Crawford A, Bohi E.S. Bonner J. 1991. Disability in Persons Hospitalised with AIDS. American Journal of physical Medicine and rehabilitation 70;2 91-95

O'Dell MW, Dillon M. 1992. Rehabilitation in adults with human immunodeficiency virus related diseases. American Journal of Medical Rehabilitation Vol. 71:3 183-190

Rehle T M. Shisana O. 2003. Epidemiological and demographic HIV AIDS projections S. Africa African journal of AIDS Research 2 ; (1) $1-8$

Roubenoff R, Schmidt H, Bairos L, Layne J, Potts E Cloutier J, Fabian D. 2002. Reduction of Abdominal Obesity in Lipodystrophy associated HIV infection by means of diet and exercise: Case report Proof and principle. Clinical Infectious Diseases; 34:390-393

Rusch M Nixon S Schilder A Braitstein P, Chan K, Hogg R S. 2004. Impairments, Activity limitations and participation restrictions: Prevalence and associations among PLWA in British Colombia; Health and Quality of life outcomes 2; www.hglo.com

TAC Annual report 1/03/04-28/2/2005)

Tehranzadeh J, Ter-Oganesyan R, Steinbach L. S. 2004. Musculoskeletal disorders associated with HIV infection and AIDS. Skeletal Radiology 33: 249-259

Tehranzadeh J, Cassady C. 1994. Misellaneous musculoskeletal conditions associated with HIV infection. In Tehranzadeh J, Steinbach L. S eds Muscoluskeletal manifestation of AIDS. St Louis: Warren h Green,: 163-174

WHO Annual Report 2004.

UNAIDS 2005 Report. 